\title{
Comparison of different optimization and process control procedures
}

\author{
Marko Reibenschuh, Franc Cus, Uros Zuperl \\ University of Maribor, Faculty of Mechanical Engineering (SLOVENIA) \\ marko.reibenschub@,uni-mb.si; franc.cus@,uni-mb.si;.uros.zuperl@uni-mb.si
}

Received February 2010

Accepted October 2010

\begin{abstract}
This paper includes a comparison of different optimization methods, used for optimizing the cutting conditions during milling. It includes also a part of using soft computer techniques in process control procedures. Milling is a cutting procedure dependent of a number of variables. These variables are dependent from each other in consequence, if we change one variable, the others change too. PSO and GA algorithm are applied to the $\mathrm{CNC}$ milling program to improve cutting conditions, improve end finishing, reduce tool wear and reduce the stress on the tool, the machine and the machined part. At the end a summary will be given of pasted and future researches.
\end{abstract}

Keywords: optimization, milling, cutting parameters, LENS

\section{Introduction}

This paper presents a comparison between two different types of machining a LENS material. The first type is a classical machining with fixed parameters such as cutting speed, feed rate, radial depth of cut, axial depth of cut and number of passes. The second type of machining has implemented a PSO optimization of cutting parameters.

In today's production industry, the need for high precision and low cost products is very high. Therefore a lot of research has been done on tool path optimization, machining performance prediction, surface roughness prediction and process 
optimization. All these researches were conducted to improve end finishing of a product. Using soft computer techniques, the input parameters (cutting speed, feed rate, radial depth of cut, axial depth of cut, number of passes,...etc.) are being optimized for a maximum end quality.

To achieve a raise in production, we need to implement a whole array of improvements and changes to our production process. The need for speed and accuracy in machining is growing every day. In a review of metal cutting analyses by Finnie (1956), it was pointed out-“Despite the large number of attempts, past and present, to analyze metal cutting, a basic relationship between the various variables is still lacking." Although this was more or less true, we could say that this statement is also valid in the $21^{\text {st }}$ century. We are on the breach; we discover and invent new materials and methods of machining every day. One of these materials is made with the LENS technology. This new method can produce composite materials. Main advantage of LENS material is the combinations of mechanical properties - we combine only the best properties to get a new material. To machine such a material, there has to be done a lot of researches and test.

At first, the technique to be used is milling. Milling is a technique, were the main feed movement is done by the tool. The end product can be stationary or, in some cases, it can also move in one or two directions. This depends on the milling centre capability. The milling centre allows us to change and control following parameters:

F - Feed rate $(\mathrm{mm} / \mathrm{min})$

$\mathrm{f}$ - Feed rate ( $\mathrm{mm} /$ tooth)

$\mathrm{N}$ - Spindle speed (rev/min)

$\mathrm{P}, \mathrm{P}_{\mathrm{m}}$ - Required power for the operation, motor power $(\mathrm{kW})$

$\mathrm{R}_{\mathrm{D}}$ - Radial depth of cut ( $\mathrm{mm}$ )

$A_{D}-$ Axial depth of cut $(\mathrm{mm})$

The main surface finish parameter $\left(R_{a}\right)$ is influenced by a correlation of parameters. Researchers use different approaches and techniques to provide a satisfactory result, this is a maximum on surface finish and a minimum on costs. To optimize and regulate such a process, major soft computing tools are used. 
The first works on the theme of optimization were published by Zadeh (1965).

\section{Choosing the right optimization methods}

Soft computing became in the early 1990's a formal computer science area of study. Earlier computational approaches and methods could model and precisely analyze only relatively simple systems. More complex systems arising in machining, biology, medicine and similar fields often remained unsolvable to conventional mathematical and analytical methods. It should be pointed out that simplicity and complexity of systems are relative, and many conventional mathematical models have been both challenging and very productive. Soft computing deals with imprecision, uncertainty, partial truth and approximation to achieve tractability, robustness and low solution cost. Components of soft computing include:

- Neural networks (NN), perceptrons,

- Fuzzy systems (FS),

- Evolutionary computation (EC), including:

o Evolutionary algorithms, genetic algorithms (GA),

o Hybrid (neuro - fuzzy, pseudo outer-product-based fuzzy neural network)

o Harmony search,

- Swarm intelligence (Particle swarm optimization - PSO, ant colony optimization - ACO),

- Ideas about probability including:

- Bayesian network,

- Chaos theory,

- Support vector machine - SVM. 
In general, soft computing techniques resemble and mimic the biological processes more closely than traditional techniques. It also parallels the ability of a biological mind to learn, observe and memorize in an environment full of parameters and other vital data. Traditionally techniques are largely based on formal logical systems, such as sentential logic and predicate logic, or rely heavily on computeraided numerical analysis (finite element analysis - FEM). Soft computing techniques are intended to complement each other. Hard computing schemes strive for exactness and full truth whereas soft computing techniques exploit the given tolerance of imprecision, partial truth, and uncertainty for a particular problem or data.

\subsection{Fuzzy systems}

Mentioned and proposed by Zadeh (1965) put forward the idea of fuzzy sets in which the elements of the set can have partial membership in the set. Many linguistic terms - words can be converted into a fuzzy set. For example, the "high feed" can be represented by a fuzzy set in which the feed values more than an upper threshold value. The value can be assigned a membership grade 1 and those lower than a lower threshold value can be assigned a membership grade 0 . Between lower and upper threshold, the feed values can have a gradual variation of membership grades from 0 to 1 . Once the linguistic variables have been converted into fuzzy sets, theoretic operations on them can be carried out. In the literature and praxis, fuzzy sets were most commonly used as follows:

- fuzzy sets help to arrive to certain decisions,

- fuzzy arithmetic,

- fuzzy logic is used for making inferences based on the input values.

\subsection{Neural nets}

Neural nets and networks are systems, based on biological systems. Imitating the primer functions of a brain, this version of soft computing techniques can acquire (learn), store and use knowledge gained from experience and learning. An artificial neural network (ANN) is capable of learning from an experimental data set or everyday data set. ANN can describe the nonlinear interaction and effects with great accuracy and success. It is composed of an input layer used to present data 
to the network, an output layer to produce ANN's response, and one, in most cases more, hidden layers in between. The input and output layers are exposed to the environment whereas the hidden layers do not have any contact with the environment. Neural networks are characterized by their topology, weight vectors, and activation function that are used in hidden and output layers. A neural network is trained with a number of data. The amount of data put in for learning is commonly very big and tested with other set of data to get to an optimum topology and weights. Once learned and trained, the neural networks can be used for event or result prediction. A brief background of neural networks is provided by Dixit (2008).

\subsection{Genetic algorithm (GA)}

Is a search technique used in computing to find approximate or exact solutions to optimization and search problems. Genetic algorithms are a particular class of evolutionary algorithms (EA) that use techniques inspired by evolutionary biology such as inheritance, mutation, selection and crossover. They incorporate the "survival of the fittest" philosophy, used by Zarei et. al (2009). In this algorithm, a point in search space is represented by binary or decimal numbers, known as string or chromosome. Each chromosome is assigned a fitness value that indicates how closely it satisfies the desired objective. A set of chromosomes is called population. A population is operated by three fundamental operations:

- reproduction (to replace the population with large number of good strings having high fitness values),

- crossover (for producing new chromosomes by combining the various pairs of parent chromosomes in the population),

- mutation (for random modification of parent chromosomes).

A sequence of these operations constitute one generation. The process repeats till the system converges to the required accuracy after many generations. The genetic algorithms have been found very useful in finding out the global minimum. Further, these algorithms do not require the derivatives of the objectives and constraints functions. 


\subsection{Simulated annealing (SA)}

Is a generic probability metaheuristic, based on the cooling process of metal during annealing, a technique involving heating and controlled cooling of a material to increase the size of its crystals and reduce their defects. It is used for the global optimization problem of applied mathematics, namely locating a good approximation to the global minimum of a given function in a large search space. For certain problems, simulated annealing may be more effective because the main goal is merely to find an acceptably good solution in a fixed amount of time, rather than the best possible solution in a larger time period.

\subsection{Ant colony optimization ( $\mathrm{ACO})$}

The ant colony optimization algorithm (ACO) is a probabilistic technique for solving computational problems which can be reduced to finding good paths through graphs and other data. This algorithm is a member of ant colony algorithms family, in swarm intelligence methods, and it constitutes some metaheuristic optimizations. Initially it was proposed by Dorigo (1996). Near-blind ants are establishing the shortest route from their nest to the food source and back. These ants secrete a substance, called pheromone, and use its trails as medium of communicating information. The probability of the trail being followed by other ants is enhanced by further deposition of pheromone by other ants moving on that same path. This cooperative behaviour of ants inspired the new computational paradigm for optimizing real life systems, which are suited for solving large scale problems with a lot of different data, mentioned by Socha and Dorigo (2008). There are different variants of ant colony optimization algorithms. These algorithms carry out three operations:

- ant-based solution construction,

- pheromone update,

- daemon actions.

Solutions are chosen probabilistically based on pheromone level. Thus, this operation forces the algorithm to search in the area of better solutions. The aim of pheromone update is to increase the pheromone values associated with good or promising solutions and decrease those that are associated with bad ones. Usually 
this is achieved by increasing the pheromone levels associated with chosen good solutions and by decreasing the pheromone values through pheromone evaporation, which basically reduces the pheromone level.

\subsection{Particle swarm optimization (PSO)}

The PSO belongs to the class of direct search methods used to find an optimal solution to an objective function (fitness function) in a search space. Direct search methods are usually derivative-free, meaning that they depend only on the evaluation of the objective function. The particle swarm optimization algorithm is simple, in the sense that even the basic form of the algorithm shows results and it can be implemented very fast. Particle swarm optimization is a population-based stochastic optimization technique developed by Kennedy and Eberhart (1995) and is inspired by the social behaviour of bird flocking or fish schooling. In PSO, each solution in search space is analogous to a member of the swarm (bird, fish) and generally called "particle". The system is initialized with population of random particles (called swarm) and search for optimum solution continues by updating generations. The fitness value of each particle is evaluated by objective function to be optimized. Each particle remembers the coordinates of the best solution (pbest) achieved so far. The coordinates of current global best (gbest) are also stored. PSO is simple to implement and does not need as much parameters to adjust as GA. In some cases, Wong and Komarudin (2008) reported that the PSO has been more efficient than GA.

\subsection{Support vector machine}

SVM is a relatively new binary classification used by Yao and Yang (2009) method with algorithm improvements. It is a margin classifier. Unlike the parameter settings of the neural networks and fuzzy-net systems, which tie the goal to reduce the errors between actual and predicted responses in the training procedure, the parameters in the SVM are used to maximize the "margin" for a credible separation of the data points. It draws an optimal hyper - plane in a high dimensional feature space. This space is determined by $w$ and $b$ - this defines a boundary that maximizes the margin between data samples in two classes - it gives a good generalization properties. The goal of employing SVM is to create predictions more robust than the methods described earlier. The main advances of SMV: 
- swift response,

- improved scalability.

Considering and analysing all these techniques, we decided to use PSO algorithm, although the last technique, SVM, is quite new and promising so we consider using it in later research.

\section{LENS technology}

LENS technology is laser engineered net shaping - a technology for repairing and building whole machine parts. This technology represents a progressive $\mathrm{HIGH}$ $\mathrm{TECH}$ technology to raise and obtain the leading role on the market. Advantages of LENS:

- production of the end product only on the digital basis,

- reduction in production time,

- computer controlling of the product formation,

- the main advantage is the combining of different materials, depending on the product function.

LENS technology provides and offers a hyper concurrence environment and it makes new possibilities on the market for new investments. It also spreads around the global market. It can be used anywhere where we need a metal product. It is used in the army for repairing the most difficult machine part (turbines), in the space and air industry, electronic industry, car industry and nevertheless in medicine industry for implants.

The production process is divided in three different parts:

- design,

- process,

- part. 
In design part, we have to design a 3D computer aided design (CAD) model. The whole model has to be transformed into an STL file format for further processing as proposed by Valentan et. al (2006). Later on, the process set up has to be done. This includes preparing the laser and all other additions. After that, the model can be built or repaired. With a multi nozzle powder delivery system, we deliver the powder to a specific point. The powder is there influenced by a powerful Nd:YAG or fiber laser. This process is similar to rapid prototyping with plastics. We build the new part layer by layer. The final step is to observe and control the finished product. In some cases the machined part has to undergo some classic machining (milling, drilling, turning,...).

\section{Combining the technology}

In our tests, we combined the new technology for part building (LENS) and the technology for optimizing the machine parameters (PSO). All the tests have been implemented on a Heller BEA 1 machining centre. The first round of tests was with the LENS part and the classical settings. Figure 1 is showing the product before the end finishing.

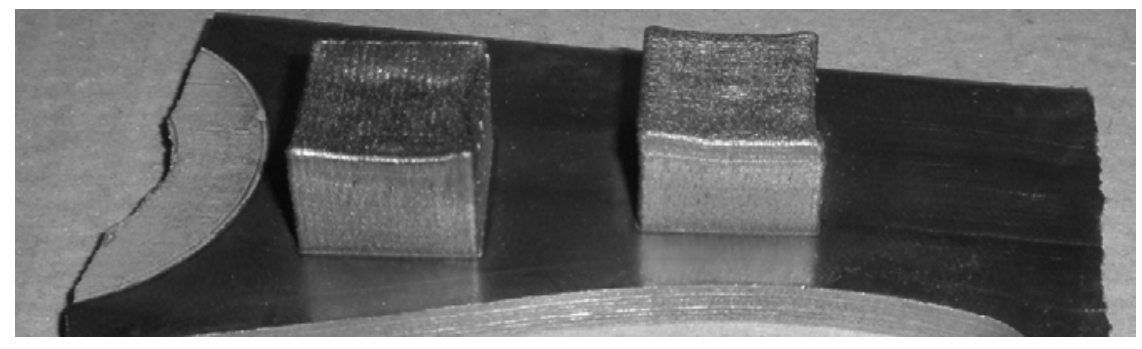

Figure 1. "Parts before end finishing $(80 \times 80 \times 60 \mathrm{~mm})$ ". Source: authors.

The parts were made on the same ground surface. The parameters are in Table 1.

\begin{tabular}{|c|c|}
\hline Part material & $\mathrm{H} 13$ \\
\hline Tool designation & CM-D20-A-C20 \\
\hline Feed rate & $430 \mathrm{~mm} / \mathrm{min}$ \\
\hline Cutting speed & $80 \mathrm{~m} / \mathrm{min}$ \\
\hline Width of cut $\mathrm{R}_{D}$ & $3 \mathrm{~mm}$ \\
\hline Depth of cut $A_{D}$ & $2 \mathrm{~mm}$ \\
\hline
\end{tabular}

Table 1. "Cutting parameters". Source: authors. 
Figure 2 shows the machined part after end finishing.
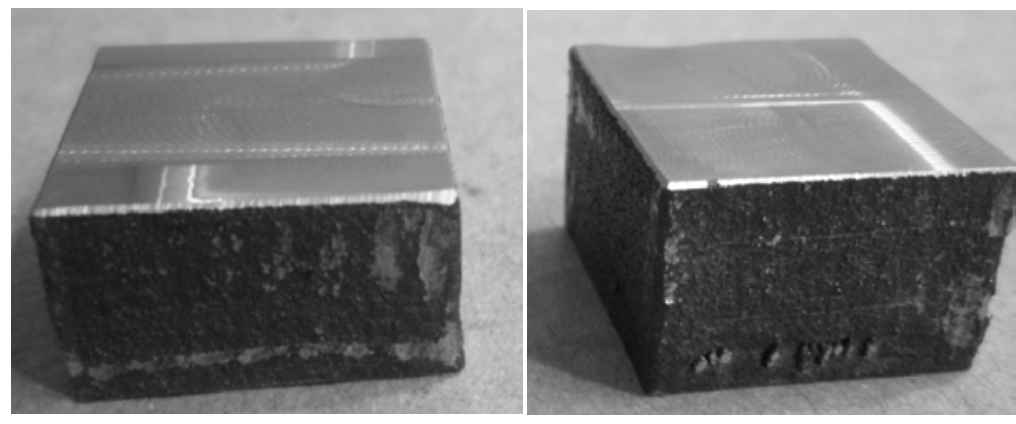

Figure 2. "Part on the left side is after machining with classical settings, part on the right shows the result with PSO optimization". Source: authors.

For the second round we used the same parameters, but we added here a PSO optimization of the tool path. This optimization is basing on the cutting force. The results are shown on Figure 2.

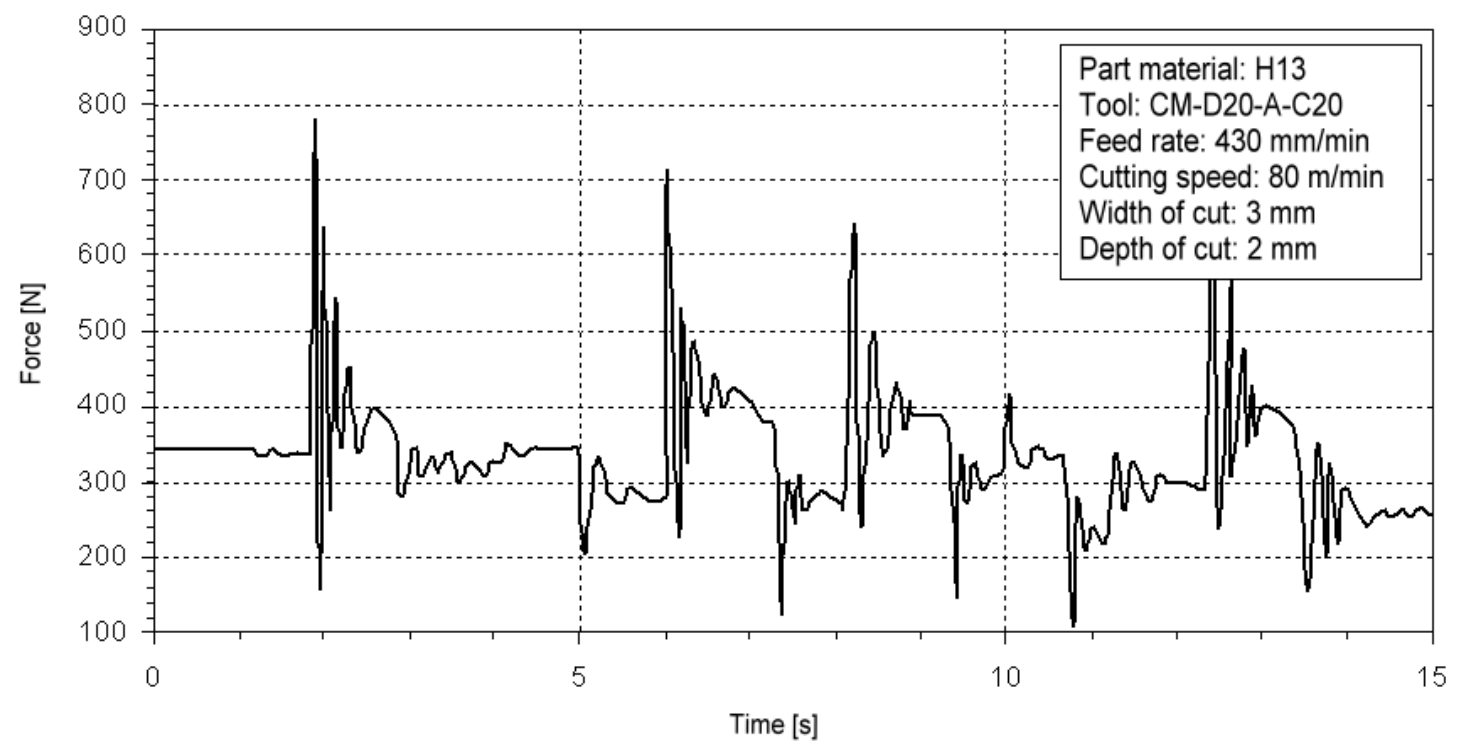

Figure 3. "Cutting force without the PSO optimization". Source: authors. 


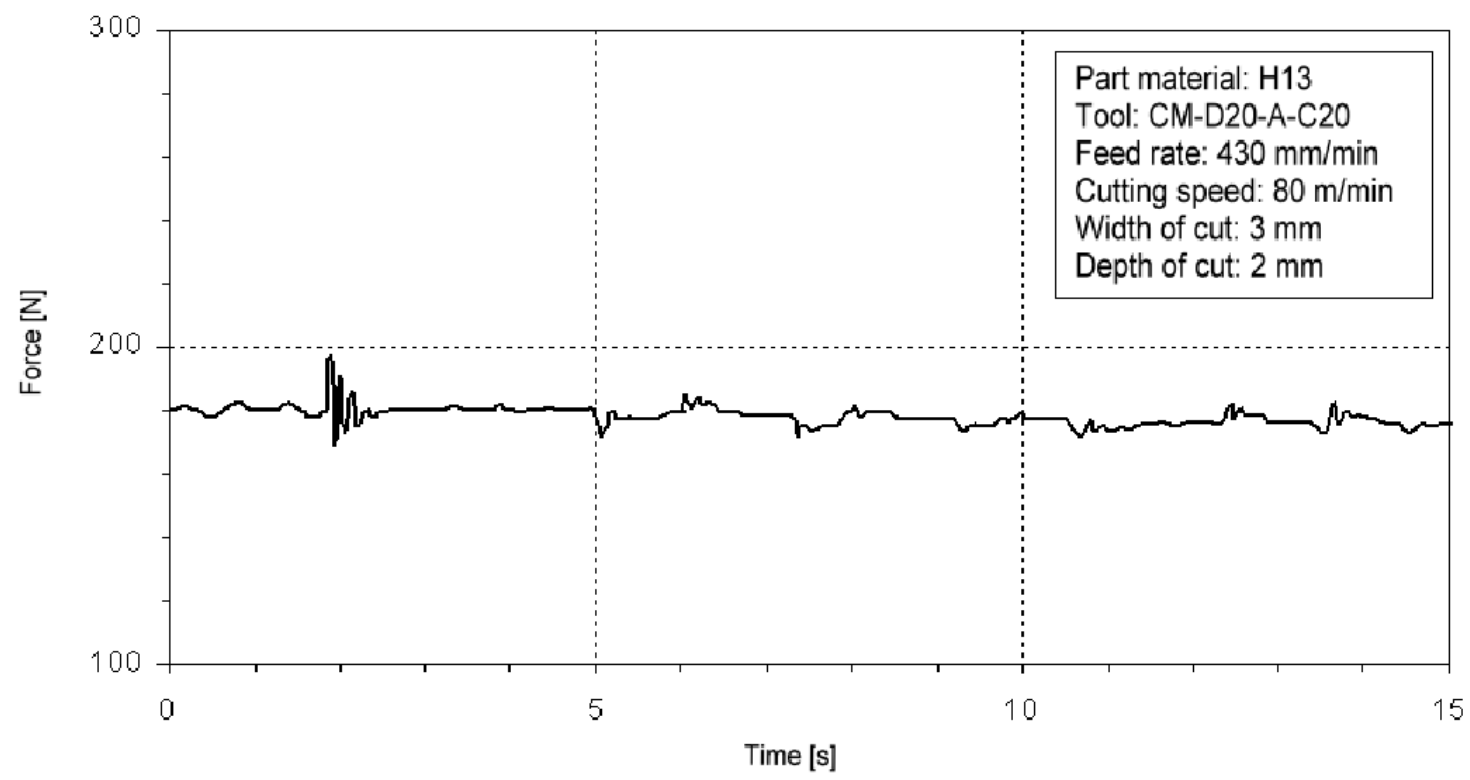

Figure 4. "Cutting force with PSO optimization". Source: authors.

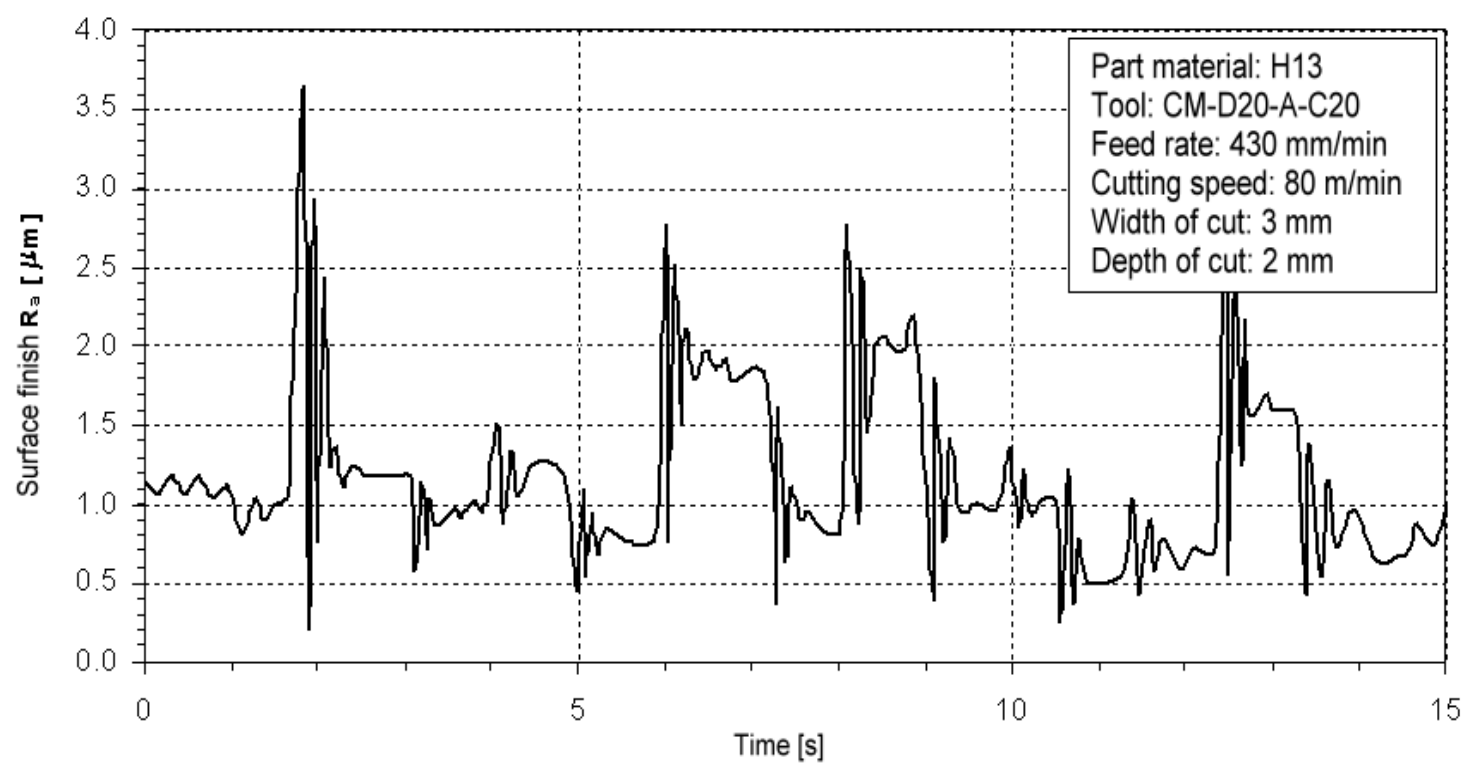

Figure 5. " $R_{a}$ before the simulation". Source: authors. 


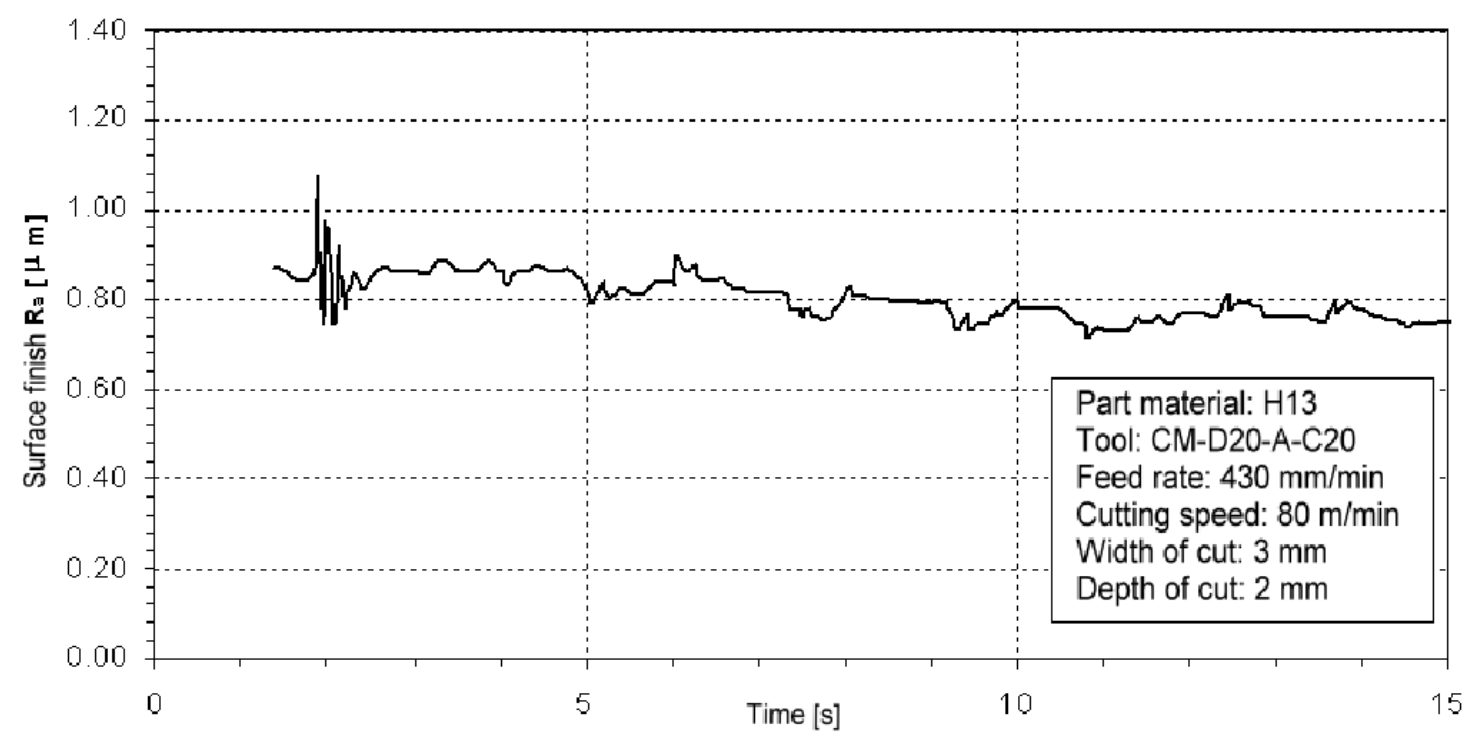

Figure 6. " $\mathrm{R}_{\mathrm{a}}$ after the simulation". Source: authors.

With the simulation and comparison we got different results. The gage for the system functionality is the difference between the desired and simulated $R_{a}$. The input data are the cutting parameters and the desired $R_{a}$.

\begin{tabular}{|c|c|c|c|c|c|c|c|c|}
\hline \multirow{2}{*}{$\begin{array}{l}\text { Sim. } \\
\text { no. }\end{array}$} & \multirow{2}{*}{$\begin{array}{c}\text { Desired surface } \\
\text { finish } R_{a}[\mu \mathrm{m}]\end{array}$} & \multicolumn{3}{|c|}{$\begin{array}{c}\text { Cutting Start parameters } \\
\text { before PSO optim. }\end{array}$} & \multicolumn{3}{|c|}{$\begin{array}{l}\text { Results of simulation after } \\
\qquad 15 \mathrm{~s}\end{array}$} & \multirow{2}{*}{$\begin{array}{l}\text { Gained } \\
\text { surface } \\
\text { finish } \\
\mathrm{R}_{\mathrm{a}}[\mu \mathrm{m}]\end{array}$} \\
\hline & & $\mathrm{F}_{\mathrm{d}}[\mathrm{N}]$ & $\mathrm{f}_{\mathrm{c}}[\mathrm{mm} / \mathrm{min}]$ & $\mathrm{n}_{\mathrm{c}}\left[\mathrm{min}^{-1}\right]$ & $\mathrm{F}[\mathrm{N}]$ & $\mathrm{f}_{\mathrm{a}}[\mathrm{mm} / \mathrm{min}]$ & $\begin{array}{c}\mathrm{n}_{\mathrm{a}} \\
{\left[\mathrm{min}^{-1}\right]}\end{array}$ & \\
\hline 1 & 0.38 & 161.4 & 357.12 & 1974 & 166.5 & 264.92 & 2443 & 0.50 \\
\hline 2 & 0.51 & 166.67 & 379.98 & 1895 & 169.9 & 282.19 & 2356 & 0.58 \\
\hline 3 & 0.64 & 172.1 & 414.53 & 1704 & 173.0 & 297.94 & 2272 & 0.66 \\
\hline 4 & 0.76 & 176.8 & 435.26 & 1563 & 175.9 & 313.94 & 2189 & 0.74 \\
\hline 5 & 0.81 & 178.5 & 430.41 & 1571 & 177.7 & 324.61 & 2134 & 0.79 \\
\hline 6 & 0.89 & 180.9 & 462.19 & 1437 & 178.6 & 329.95 & 2108 & 0.81 \\
\hline 7 & 1.02 & 184.7 & 485.14 & 1377 & 181.6 & 350.52 & 2007 & 0.91 \\
\hline 8 & 1.14 & 188.1 & 507.75 & 1270 & 183.9 & 355.60 & 1925 & 0.99 \\
\hline 9 & 1.27 & 191.2 & 539.24 & 1116 & 185.7 & 381.25 & 1880 & 1.05 \\
\hline 10 & 1.52 & 196.8 & 592.58 & 825 & 195.2 & 411.99 & 1716 & 1.18 \\
\hline
\end{tabular}

Table 2. "Results of the simulation". Source: authors. 
Table 2 shows the demands and results of the test - simulation. 10 different simulations were done. The results in Table 2 confirm that the system with PSO optimization is superior to the classical one. This system is also successful at the end finishing. The system response slows down if we go over a certain value; in our case is this value at $1.18 \mu \mathrm{m}$. We have managed to reduce the cutting force and improved the surface finish. Although the values for revelation per minutes are rising, this should be no problem because the new tools are designed for high speed cutting and higher revelations.

From Figure 3 to Figure 6, we can see, that we can on the basis of the cutting force predict the quality of end finish, respectively the surface finish.

\section{Process control procedures}

The purpose of the process control procedure is to ensure that activities are conducted in a planned and systematic manner through the implementation of a process. Two activities are vital for this job:

- process monitoring and,

- process regulation.

Different authors as Chandrasekaran et. al (2010) are proposing different methods for the problem of regulating a process. Some prefer ACO over GA as Wong and Komarudin (2008) because of its superior performance. Others like Baskar and Asokan (2005) see the superior method in PSO because of better results and its versatility in usage. In a paper from Chandrasekaran et. al (2010) that reviews a lot of the research done till this day in this field, it is said, that NN are often used at drilling and turning, whereas for milling and grinding fuzzy sets are used.

In our test, the process control procedure was done with the help of pressure sensors (Kistler 9255) for detecting the corresponding cutting force of the tool. This solution works by using sensors to monitor key stages of the process. The sensors are connected to a PC connected gathering data card (PC-MIO-16E-4). This unit produces a continuous flow of measurement results. A software system handles the results and either regulates the process automatically or helps the production operator to make required adjustments.

As mentioned above, two different types of regulation can be implemented: 
- a fully automated, where we put in the values for certain parameters and program what to do if those parameters are exceeded or not reached,

- or partly automated, where we have during the process of regulation control over certain parameters.

In our case a partly automated process control procedure was used. For this purpose a special program was written and PSO algorithm was implemented into it.

For the process to be fully automated, more sensors and programs need to be added. For comparison the automotive industry - the process is overseen with different sensors:

- motion sensors,

- vibration sensors,

- pressure sensors,

- temperature sensors,

- etc...

To combine all the gathered data, a big, powerful and reliable system is needed. In nowadays production, all the expenses are held to a minimum. Such a big system costs a lot of resources, on the other hand, a smaller one, partly automated system costs $1 / 100^{\text {th }}$ of the big one. This is also one of the main reasons that we decided to develop our own system.

For our needs, the system is fast enough and enough powerful to implement it even on the newest production centres.

\section{Conclusion}

With the comparison of two different approaches, we came to the conclusion, that we can implement soft computer techniques (optimization) also to new technologies and materials. With the help of optimization tools we can reduce production time, improve end finishing by raising the quality and in the same time produce more parts. Also the process control procedures are very important, to assure proper function of the machining centre.

For further work we would suggest further tests and implementation of new techniques. One of them is emerging on the horizon. The technology is new and it 
has to prove its potential - DNA computers - smaller and faster, for faster data handling and larger applications.

\section{References}

Baskar, N., \& Asokan, P. (2005). Optimization of machining parameters for milling operations using non-conventional methods. International Journal of Advance Manufacturing Technology, 25, 1078-1088.

doi: 10.1007/s00170-003-1939-9

Chandrasekaran, M., Muralidhar, M., Murali Krishna, C., \& Dixit, U.S. (2010). Application of soft computing techniques in machining performance prediction and optimization: a literature review. International J ournal of Advance Manufacturing Technology, 46, 445-464.

doi: $10.1007 /$ s00170-009-2104-x

Dixit, P.M. (2008). Modeling of metal forming and machining processes: by finite element and soft computing methods. Springer: London.

Dorigo, M. (1996): The ant system: optimization by a colony of cooperating agents. IEEE Transnational System Management Cybernetics, 26, 1-13.

Finnie, I. (1956). Review of the metal-cutting analysis of the past hundred years. Mechanical Engineering, 78, 715-721.

Hsueh, Y.-W., \& Yang, C.-Y. (2009). Tool breakage diagnosis in face milling by support vector machine. J ournal of Materials Processing Technology, 209(1), 145152. doi: 10.1016/j.jmatprotec.2008.01.033

Kennedy, J ., \& Eberhart, R. (1995). Particle swarm optimization. Proceedings of the IEEE International Conference on Neural Networks (ICNN'95), Perth, Australia.

Socha, K., \& Dorigo, M. (2008). Ant colony optimization for continuous domain. European J ournal of Operation Research, 185, 1155-1173.

doi: 10.1016/j.ejor.2006.06.046

Valentan, B., Brajlih, T., Drstvensek, I., \& Balic, J. (2006). Evaluation of shape complexity based on STL data. Journal of achievements in materials and manufacturing engineering, 17, 1-2. 
Wong, K.Y., \& Komarudin (2008). Parameter tuning for ant colony optimization: a review. Proceedings of the international conference on computer and communication engineering 2008, Kuala Lumpur, Malaysia.

Zadeh, L.A. (1965). Fuzzy sets. Information and Control, 8(3), 338-353.

doi: 10.1016/S0019-9958(65)90241-X

Zarei, O., Fesanghary, M., Farshi, B., Jalili, S.R., \& Razfar, M. R. (2009). Optimization of multi-pass face-milling via harmony search algorithm. Journal of Materials Processing Technology, 209, 2386-2392.

doi: 10.1016/j.jmatprotec. 2008.05.029

(c) Journal of Industrial Engineering and Management, 2010 (www.jiem.org)

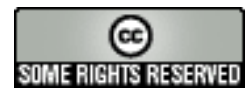

\footnotetext{
Article's contents are provided on a Attribution-Non Commercial 3.0 Creative commons license. Readers are allowed to copy, distribute and communicate article's contents, provided the author's and Journal of Industrial Engineering and Management's names are included. It must not be used for commercial purposes. To see the complete license contents, please visit http://creativecommons.org/licenses/by-nc/3.0/.
} 